\title{
La responsabilidad civil de los Bancos en el Servicio de Cajas de Seguridad
}

Juan EsPINOZA EsPINOZA

\section{Premisa}

El servicio de cajas de seguridad en sus orígenes no era de competencia exclusiva de las instituciones bancarias. En efecto, los horrea de la antigua Roma, que eran grandes almacenes fortificados de propiedad imperial o de los privados, constituyeron sus más remotos antecedentes históricos $^{1}$. No obstante ello, el servicio de cajas de seguridad, así como lo conocemos en la actualidad, es relativamente reciente.

Inicialmente sólo las compañías privadas se encargaban de ofrecer este servicio y, paradójicamente fue mucho tiempo después que los bancos se convirtieron en sus proveedores exclusivos, aunque este servicio haya sido definido «el menos bancario de todos»². La razón de ello está en el hecho que las empresas bancarias, vista su solidez económica por excelencia, pueden disponer de la tecnología más avanzada, en lo que se refiere a las medidas de seguridad, idónea a neutralizar las causas generadoras de eventuales pérdidas o robos.

El legislador italiano ha preferido eludir la definición de este contrato ${ }^{3}$ limitándose, a través de la lectura del combinado dispuesto del art. 1839 c.c. y del Art. $3^{\circ}$ primer párrafo, de las Normas para el servicio de las cajas de seguridad comprendidas en las Normas bancarias uniformes

1 Molle, "I contratti bancari", en Tratt. di dir. cip. e comm. Cicu-Messineo. XXXV, tomo I, 4ta. de., Milano, Giuffré, 1981, 787. El autor sostiene que este precedente es importante a los efectos de la calificación de la relación jurídica, en cuanto se revela como una figura extraña a la del depósito (MOLLE, Op. cit. 788).

2 La expresión es de CASTRO NUOvo, "Tra rischio e caso fortuito. La responsabilità da cassette di sicurezza", en Le operazioni bancarie, a cura de Portale, I, Giuffrè, Milano, 1978, 479.

3 Molle, Op. cit. 796-797. 
(nbu), a trazar los confines de las responsabilidad del banco con respecto el usuario "por la idoneidad de la custodia de los locales y por la integridad exterior de la caja, salvo el caso fortuito». Según la reciente orientación jurisprudencial parecería que asegurar tal integridad externa fuese una fórmula eufemística, porque siempre, en el momento patológico de la relación usuario-banco, en el caso que exista hurto o robo, a los efectos de valorizar al resarcimiento al cual está obligado el banco en cuanto sujeto responsable, deberá cuantificarse el contenido interno de la caja. Se crea una suerte de círculo vicioso cuya fórmula podemos enunciar de la siguiente manera: No conozco lo que está dentro de la caja y únicamente asumo la custodia de lo que está afuera (local, instalaciones) pero, cuando no haya cumplido con la obligación a la cual me he comprometido, respondo sólo por una suma mínima (que es acordada en base a los Art. $2^{\circ}$ y $3^{\circ}$, segundo párrafo, de las nbu).

Cuando se promueva una acción judicial en contra del banco, sobre el dañado gravará la carga de probar cuál ha sido el efectivo contenido de la caja. Su cumplimiento, de éxito asaz incierto, constituye una suerte de probatio diabólica, una "lotería forense» ${ }^{4}$ que tendrá el efecto de favorecer o al usuario o al banco. Tanto en el momento fisiológico como en el momento patológico de la relación que tiene origen en el contrato en objeto, existen diversos derechos e intereses que están en conflicto entre si, entre otros, el secreto del contenido de la caja, la limitación de la responsabilidad del banco y la tutela del usuario en su status de consumidor .

La dificil tarea que le corresponderá absolver a la jurisprudencia, es aquella de delimitar e interpretar los modelos jurídicos abiertos que ha diseñado el legislador, en manera tal de conciliar estos intereses.

\section{Fundamentos normativos: réplica y duplica entre la A.B.I. y la jurisprudencia}

El código civil italiano de 1942 regula parcialmente el servicio bancario de las cajas de seguridad en los artículos $183^{\circ}$ (límites de la responsabilidad del banco hacia el usuario), $1840^{\circ}$ (apertura de la caja por parte de los titulares y de los legitimados en caso de muerte) y $1841^{\circ}$ (apertura forzada de la caja). En 1954, la «Asociación Bancaria Italiana (A.B.I)

4 Expresión usada por ALPA, Responsabilità civile e danno. Lineamentie questioni, Il Mulino, Bologna, 1991, 528. 
había preparado un modelo de contrato, cuyo artículo $16^{\circ}$ prevé, entre otros aspectos, que «el valor máximo del contenido de la caja queda, para todos los efectos, convencionalmente fijado en ... liras, salvo que, de acuerdo con el usuario, se establezca un importe superior, contra el pago de la contraprestación determinada por la hacienda de crédito.

En el caso en el cual, por cualquier razón, la hacienda fuese obligada a un resarcimiento en beneficio del usuario, ésta sólo reparará el daño objetivo, quedando excluida toda apreciación del valor afectivo o teniéndose en cuenta lo dispuesto en el párrafo precedente».

Sobre la interpretación de tal cláusula se han formado dos corrientes de pensamiento a nivel doctrinal y jurisprudencial. Una orientación que entendía una función limitativa del objeto de contrato ${ }^{5}$, en cuanto, no teniendo el banco el derecho a conocer el contenido de la caja de seguridad, le es conferida la facultad de convenir con el usuario el valor máximo de las cosas que este podrá introducir en la misma, con la finalidad de establecer el canon al cual está obligado el usuario. En consecuencia de ello «el cliente (...) que introduce en la caja completivamente valores superiores a aquellos declarados, viola el contrato y no puede pretender el cumplimiento del banco fuera del contrato violado por él mismo» ${ }^{6}$.

Según una diversa escuela de pensamiento, la cláusula en objeto era entendida como limitativa de responsabilidad?. En tal sentido se ha pro-

5 En doctrina, GianNaTTASIO, "Limitazione della responsabilità della banca nel servizio delle cassette di sicurezaa", en BBTC, 1956, II, 441, que afirmaba que: "Si el usuario ha dado a sus bienes el valor de 100 , no podrá después pretender un resarcimiento mayor de tal cifra, que importaria un enriquecimiento injusto con daño al banco" (cit. 444); MolLE, "Caso fortuito e responsabilità della banca nel servizio di cassette di sicurezza", en BBTC, 1976, II, 173; CAPOBIANCo, "Le cassette di sicurezza", en BBTC, 1955, I, 102; ToNoo M., Contratti bancari, Roma, 1967, 265; Scordino, I contratti bancari, Napoli, 1965, 469; CiuchiNI, "Il servizio bancario delle cassette di sicurezza", en Enciclopedia della banca e della borsa, Roma-Milano, 1967, II, 151 e 173 . En jurisprudencia, TRIB. Frrinze, 11.03.71, en Foro Pad., 1972, I, 1, 232, con nota de ALPA, Caso fortuito e responsabilitì della banca per il servizio delle cassette di sicurezza, y también en Giurisprudenza di diritto privato, I, Giapichelli, Torino, 1991, 372. En esta sentencia se acoge la línea de pensamiento de Giannatrasio del «injusto enriqueciniento con daño al banco".

6 Moll., Op. cit., 176.

7 CAS., 05.07.56, 11. 2451, en BBTC, II, 1956, 438, sanciona que el Art. $13^{\circ}$, párrafo 3 , del reglamento para el servicio de las cajas de seguridad del Banco de Nápoles, que reproducía el Art. $16^{\mathrm{Q}}$ nub, debe considerarse inválido, porque está «en contraste con las disposiciones del segundo párrafo del Art. $1341^{\circ}$ c.c., que excluye, en todo caso, la eficacia de las condiciones particularmente onerosas, como aquellas que establecen 
nunciado la Corte de Casación, que en la sentencia № 1129 del 29.03.76, ha considerado que el Art. $16^{\circ} \mathrm{nbu}$, forma parte de lo previsto por el Art. $1229^{\circ}$ c.c., con la inevitable consecuencia que dicha cláusula debe considerarse nula en los casos de responsabilidad por dolo o por culpa grave ${ }^{8}$. Como inmediata respuesta, la A.B.I., mediante circular del 28.09.76, ha considerado oportuno modificar las normas bancarias uniformes. La previsión del viejo art. 16, primer párrafo, ha sido trasladada, con algunas modificaciones, al actual Art. $2^{\circ}$, que recita: «el uso de la caja es concedido para la custodia de cosas de valor total no superior a ... liras. Por tanto, el usuario se obliga a no conservar en la cajas cosas que tengan un valor total superior a dicho importe de ... liras». El segundo párrafo del abrogado Art. $16^{\circ}$, en cambio, ha encontrado sitio, prácticamente sin modificación, en el nuevo Art. $3^{Q}$ nbu.

Hasta hace poco, doctrina ${ }^{9}$ y jurisprudencia ${ }^{10}$ han mantenido un

limitaciones de responsabilidad, a favor de quien las ha dispuesto, si éstas no son específicamente aprobadas por escrito".

8 Esta es la motivación de la sentencia: «la inserción del art. 16 de las normas bancarias uniformes en un contrato de utilización de cajas de seguridad, no altera en ninguna manera el objeto del negocio, que continúa siendo típico, previsto por el Art. $1839^{\circ}$ c.c. (textualmente reproducido en el primer párrafo del mismo Art. $16^{\circ}$ ). La determinación convencional del valor máximo, por atribuirse al contenido de la caja, no está vinculada a alguna restricción o dilatación de las obligaciones deducidas en el contrato, ya que no importa ni para el cliente la prohibición de introducir en la caja cosas de valor, ni para el banco un diverso comportamiento en las obligaciones que le corresponden" (en BBTC, 1976, II, 179). Este criterio ha sido confirmado en CAS., 03.11 .89 , No 4604, en Foro it., 1990, I, 1290, con nota di Consintino, Il contratto di servizio delle cassette di sicurezza: clausola di limitazione della responsabilità della banca dichiarazioni di valore; en NGCC, 1990, I, 320, con nota di PuHALI y en BBTC, 1991, II, 508 .

9 BeNATTI, Le clausole di esonevo da responsabilità nella prassi bancaria, a cura de Portale, Milano, 1978, I, 144; Cabella Pisu, "Le clausole di esonero da responsabilità, en Tratt. Rescigno, 9, 233; Chrrai, Cassette di sicurezza, en Digesto. IV ed., Torino, 1988, 21; Consentrovo, Op. cit.; Mollt, I contratti bancari, cit., 823; G.B. FERR, "Tipicità negoziale e interessi meritevoli di tutela nel contratto di utilizzazione di cassette di sicurezza", en Riv. dir. comm., 1988, I, 345; Amoroso, "Contratti bancari e condizioni generali di contratto", en Le condizioni generali di contratto, a cura de BIANCA, 1981, 101, entre otros.

10 Así, TRIB. Roma, 27.01.82, en BBTC, II, 1984, con nota de Salanitro; Trib, Roma, 26.11.87, en Il corr. giur., 1988, 494, con nota de Mariconida; Trib. Roma, 27.04.90, en BBTC, II, 1991, 507; AP, Roma, 06.03.90, ipi; AP, Roma, 28.09.90, en Giust. civ., 1990, I, 2959 y AP, Roma, 17.12.91, en Giust. civ., 1992, I, 1582, Comentario particular merece el Trib. NAPol, 16.03.84, en BBTC, 1985, II, 506; también en Bancaria, 1985, I, 236, con nota de Gabrielli, Cassette di sicurezza e clausola penale, que ha resuelto el problema en estos términos: "la cláusula que prevé el 
comportamiento mas bien uniforme al interpretar las cláusulas del texto mencionado como limitativas del objeto del contrato. En particular, se ha sostenido que el banco, para cumplir con sus obligaciones, debe preparar medidas de seguridad idóneas a afrontar las singulares situaciones "a riesgo» y que, en consecuencia, sería lícito establecer límites máximos para el valor de los bienes que la caja está en grado de custodiar ${ }^{11}$. En todo caso, cuando el banco considere que el cliente no cumple con sus propias obligaciones, depositando bienes de valor superior a aquel previsto contractualmente, este bien podría actuar en aplicación del Art. $4^{\circ}$ nbu, que consiente la apertura forzada de las cajas ${ }^{12}$. En esta relación

valor de las cosas a introducirse en la caja de seguridad, no está finalizada a limitar la responsabilidad del banco, sino a establecer una relación de proporción entre tal responsabilidad y el monto del canon o a predeterminar la prestación resarcitoria, vinculándola a un valor convencionalmente pactado». Esta orientación jurisprudencial que identifica la cláusula penal con aquella de exoneración de responsabilidad, es solitaria. Se ha observado su manifiesta diferencia, dado que estas últimas adebilitan el vínculo obligatorio", en tanto que las primeras refuerzan el vínculo contractual (Garrielli, Op. cit., 24l).

11 Así, Ferrfttı, quien afirma: "Es por tanto del todo admisible que el banco establezca en casos similares una prohibición para el usuario de introducir bienes más allá de un cierto valor en la caja. Tal prohibición no subyacería ni a la aprobación específica por escrito ex Art. $134 \mathrm{I}^{\circ}$, en cuanto no sería una cláusula general vejatoria para el cliente, ni a la sanción de nulidad prevista por el Art. $1229^{\circ}$, en cuanto constituiría una cláusula de exoneración de la responsabilidad del banco por dolo o culpa grave" ( $\mathrm{La}$ responsabilità contrattuale della banca per la manomissione delle cassette di sicurezza, en Responsabilità contrattuale de extracontrattuale delle banche, Giuffrè, Milano 1986, 186). En este sentido Castronuovo sostiene que el usuario, en el caso de haber depositado bienes en la caja con un valor superior al pactado y que haya sobrevenido una pérdida, la solicitud de un pretendido resarcimiento constituiria un «penire contra factum porque el mayor daño sería debido al incumplimiento de la obligación accesoria del cliente mismo» (cit., 496).

12 Ferretti, Op. cit., 187. El primer párrafo del Art. $4^{\circ}$ nbu recita: «queda prohibido al usuario introducir en la caja cosas que puedan constituir peligro de cualquier género o que puedan ocasionar daño, perjuicio o disturbio". El autor sostiene que el hecho de colocar bienes de valor superior al pacto hace que éstos sean cosas "que pueden constituir peligro" para la seguridad del cavean. Estamos de acuerdo con la lectura sistemática de las normas bancarias uniformes, pero disentimos de la interpretación del autor en cuanto el hecho que nos mueve a comentario, al máximo puede "ocasionar daño o perjuicio", dado que el artículo ha sido diseñado frente a la posibilidad de colocar cosas que puedan ser in re ipsa «peligrosas» para el banco, en cambio la disyunción después de esta previsión puede comprender situaciones que no se identifican en este concepto de "peligro", como por ejemplo el hecho de depositar cosas de valor superior a aquel acordado contractualmente. En sentido crítico, PAPANTIPelletifr, quien afirma que se trata de un forzamiento legislativo (Cassette di sicurezza $e$ responsabilità del banchiere, GIUfFrè, Milano, 1988, 127). 
banco-usuario reviste notable importancia el respeto del principio de la buena fe, tanto de parte del banco a que no imponga cláusulas que atenten contra los intereses de los clientes, como por parte de los usuarios, a los cuales no está permitido introducir en la caja bienes con un valor superior al establecido ${ }^{13}$.

Una parte de la doctrina critica el recurso al criterio de la proporción entre el canon y el límite del resarcimiento, en cuanto «una limitación de responsabilidad que varíe con el cambio de canon no muta por ello de naturaleza, no deja de ser limitación, sino sufre sólo una adecuación

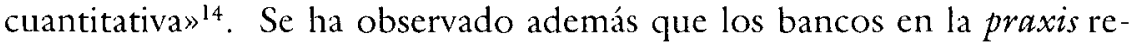
lativa al servicio de las cajas de seguridad, a menudo estipulan un contrato de seguro por una eventual responsabilidad civil, cuyo costo está incluido en el canon que debe pagar el usuario. Tal praxis, puede ser considerada en conflicto con el principio de la transparencia. Cuando se considere que tal débito no deba ser necesariamente comunicado al usuario, en virtud de la accesoriedad del contrato de seguro, que estaría incluido en el objeto del contrato principal, relativo al servicio de las cajas de seguridad, el artículo $2^{\circ}$ nbu sería plenamente válido. En caso contrario, cuando haya una obligación de información respecto del mencionado costo adicional, «entonces la utilización por obra de la empresa de una parte del precio para cubrir los costos de seguro sin que se dé noticia al consumidor o usuario, significa cambiar el contrato a finalidades sustanciales distintas de aquellas por las cuales el contrato se constituyó, en el plano de la objetividad formal y de la conciencia de una de las partes, $(\ldots)$ : se cae en aquello que se puede definir abuso del tipo contractual $»^{15}$. Por ello se ha concluido que el Art. $2^{\circ}$ nbu estaría atacado de nulidad ex Art. $1418^{\circ}$, primer párrafo ${ }^{16}$. Esta tesis ha sido tachada de "artificiosidad manifiesta»" ${ }^{17}$, por cuanto se sostiene, que los bancos no aseguran el servicio del clientes individualmente considerado, sino en la totalidad, distribuyendo el costo entre todos los usuarios y dándoles cuenta a los mismos, de conformidad con el Art. $1^{\circ}{ }^{\circ}$ nbu $^{18}$.

\footnotetext{
13 Así. Ferretti, Op. cit., 188.

14 Castronuovo, Op. cit., 493.

15 Castronuovo, Op. cit., 498.

16 Castronuovo, Op. cit., 500.

17 MolLe, In tema di responsabilità della banca nel servizio delle cassette di sicurezza, en BBTC, 1978, II, 460.

18 Cuyo primer párrafo prescribe que: «El usuario está obligado a pagar anticipadamente los canones establecidos por la hacienda de crédito para el uso de las cajas de seguridad y dados a conocer mediante aviso expuesto en los locales de la oficina donde se encuentran las cajas de seguridad, asi como de los gastos y las cargas causados por
} 
Entre aquellos que contestan la mencionada tesis, hay quien, partiendo de la distinción entre tipo (calificación de la operación en los esquemas abstractos positivamente disciplinados) y causa (valoración de la licitud de la finalidad completa perseguida por las partes) llega a la conclusión que la noción de «abuso de tipo contractual» no sea causa de nulidad, "no siendo aplicable en el ordenamiento ninguna norma imperativa que sancione en tal sentido la desviación de los modelos abstractos fijados en el código» ${ }^{19}$.

Hay otro que advierte que la cláusula 3 , párrafo 2 nbu, es una suerte de "máscara" bajo la forma de la denominada delimitación del objeto ${ }^{20}$. En efecto, "la única cláusula en concreto de la cláusula del examen, consiste en la delimitación de la responsabilidad del banco» ${ }^{21}$, en consecuencia, el contrato de las cajas de seguridad tiene la causa ilícita por contrariar a la norma imperativa (Art. $1229^{\circ}$ c.c.) y es atacado de nulidad, $e x$ Art. $1343^{\circ}$ c.c. ${ }^{22}$.

Tomando como punto de partida el aislado dictum del Tribunal de Nápoles, contenido en la sentencia 16.03.84, hay quien prospecta una relación entre cláusula penal y cláusulas limitativas de responsabilidad, afirmando que la previsión de un techo máximo de resarcibilidad del daño, incluida en los contratos de cajas de seguridad epuede ser considerada, por voluntad (implícita) de las partes, una cláusula de liquidación convencional y preventiva del daño» ${ }^{23}$. En el ámbito del mismo orden de ideas se colocan tres hipótesis de conclusión de contrato de cajas de seguridad:

a) El cliente no tiene interés en negociar el contrato y hay, por tanto, una suerte de contratación pasiva, en las cuales se aceptan sic et simpliciter, las cláusulas predispuestas por la $\mathrm{ABI}$.

b) Ambas partes tienen el interés de negociar el contrato, interpretando y reelaborando el Art. $2^{\circ}$ nbu como cláusula penal.

c) Las partes se «desvinculan» del Art. $2^{\circ}$ nbu mediante renuncia al secreto del contenido de las cajas de seguridad.

el contrato; renovándose el contrato, aún por prórroga tácita, después de una modificación del canon, se debe el canon nuevo".

19) SHPt, Incidenza del «fortuito» nei contratti di cassetta di sicureza, en BBTC, $1991, I I ; 776$.

20 Papanti-Pelletitr, Op. cit., 124 e 173.

21 Papanti-Phlletiler, Op. cit., 137.

22 Papanti-Pelletilir, Op. cit., 170.

23. Así AмATo. La responsabilità della banca per il servizio delle cassette di sicurezza: gli ultimi sviluppi, en Resp. Civ. e Prev., 1990, 780. 
La ventaja de los contratos sub b) está en el hecho que se puede prescindir de la prueba del daño, dado que la cláusula penal operaría en su función resarcitoria ${ }^{24}$. Se considera, sin embargo, que los contratos sub c), constituyan «la solución más equitativa ${ }^{25}$ en cuanto están dirigidos hacia un resarcimiento integral. Este planteamiento entra en manifiesto contraste con la praxis bancaria, en cuanto, al interior de cualquier entidad bancaria existe una normativa en la que se prevé la prohibición de aportar modificaciones en las cláusulas contractuales contenidas en las normas uniformes. El servicio de las cajas de seguridad se caracteriza, además de la garantía - vale la pena recordarlo- de seguridad, también por aquella del secreto, por tanto, si el banco contrata con los clientes teniendo conocimiento del contenido de las cajas, lejos de ser prisioneros de las tipologías, admitiremos que estamos esbozando la naturaleza de cualquier otro contrato menos que el de caja de seguridad.

Hay quien critica las tesis de aquellos que valorizan los Arts. $2^{\circ}$ y $3^{\circ}$ nbu basándose en una formulación abstracta, «sin poner atención sobre el contenido concreto de los contratos individualmente considerados concluidos entre el banco y el usuario en los cuales las mismas cláusulas están incluidas ${ }^{26}$. Se sostiene, en particular, que la valorización debería ser hecha caso por caso, sin que el uso del instrumento de la tipicidad, en mérito al cual tanto discuten los teóricos que estudian este contrato, sea un freno a la calificación de la relación jurídica entre las partes. Moviendo también de la exigencia de reserva a la cual está obligado el banco respecto al contenido de las cajas, "debe considerarse admisible (y obligada), la determinación del objeto» ${ }^{27}$, que se realiza a través de la delimitación de los bienes que pueden ser depositados en la caja, según la real intención de los contrayentes. También en este planteamiento el principio de la buena fe juega un rol decisivo en la fisiología de la relación contractual. Se ha propuesto distinguir las cláusulas de limitación de responsabilidad (cuando el banco no ofrece al usuario la responsabilidad de elevar el quantum del resarcimiento) de aquellas de determinación del objeto del contrato (cuando, en cambio, se ofrezca dicha posibilidad, prospectando «al cliente un equitativo sistema de graduación del canon en relación a los bienes depositados y al consiguiente resarcimiento» ${ }^{28}$ ). A

\footnotetext{
24 AMAT(), Op. cit., 790.

25 AмATo, Op. cit., 791.

26 FESTI, Le norme bancarie uniformi sul servizio delle cassette de sicurezza e la responsabilità della banca, en Contratto e impresa, CEDAM, Padova, 3, 1991, 1226.

27 FrsTI, Op. cit. 1228-1229.

28 FisT1, Op. cit. 1242.
} 
diferencia de la doctrina dominante, que estigmatiza el comportamiento de los bancos, se ha propuesto, como causa de invalidez del contrato del servicio de las cajas de seguridad, la responsabilidad de ambas partes: del banco, cuando fije un límite de partida irrisorio y de los usuarios, cuando acepten dicho límite con la finalidad de pagar un canon más bajo.

\section{La reciente orientación jurisprudencial interpretativa de las cláusulas predispuestas por la A.B.I.}

El Tribunal de Roma con sentencia de fecha 30.05.87, ha afirmado que es extraña al dictado normativo la posibilidad de limitar contractualmente «la naturaleza y el valor de los objetos a colocarse» en las cajas, siendo la posición del usuario, que se compromete en estos términos, «excesivamente penalizada» respecto a la del banco. En consecuencia, aplicando los criterios de valorización ex Art. 1322을 párrafo 2 c.c., ha declarado nula la cláusula 2 nbu, por que contrasta con las exigencias de un equilibrado contemperamiento de los intereses ${ }^{29}$. El mismo Tribunal, con sentencia 08.07.87, № 9386 $6^{30}$, ha nuevamente declarado la nulidad del artículo $2 \mathrm{nbu}$, sin hacer la mínima referencia a la naturaleza del este, vale decir si es limitativa del objeto del contrato o del ámbito de responsabilidad del banco ${ }^{31}$.

29 TRIB. Roma, 30.05.87, en Giust. civ., 1988, I, 273, con nota de Santarsierre, Clausola di limitazione dei valori da custodive in cassette di sicurezza e mancanza di tutela secondo I'ordinamento giuridico, quien sobre este decisium opina que: «Ha llegado el turno esperado por los contrayentes más débiles, se remueve acquel estado de «inferioridad contractual» de una parte (cit., 276).

31) TRIB. Roma, 08.07.87, n. 9386, en $I l$ corr. giur., 1987, 1274, con nota crítica de Mariconisa y en Giur. it., 1989, 694, con nota di Nicolal y otra de Colonvello, Art. $16^{0}$ dell Norme bancarie uniformi: limitazione di responsabilità o limitazione dell'oggetto?. Esta última, en sentido crítico afirma que: «el haber predipuesto los límites a las cosas a custodiar (...) no quiere decir desnaturalizar el contrato (cuya causa es siempre la custodia), sino cautelarse en contra de pretensiones de resarcimiento ilimitado; la introducción de un límite con la finalidad de cuantificar las prestaciones del banco, incide, por tanto, sobre el objeto del contrato y no sobre la causa» (cit., 710).

31 "Puesto que la inclusión de dicha cláusula en el contrato, da vida a un contrato atípico, en sus alcances debe ser operada la valoración del mérito de tutela a los efectos del art. 1322, segundo párrafo, c.c. Teniendo en cuenta la calidad de las partes contrayentes y de los costos que importan la preparación de las cajas de seguridad, una convención por la cual el banco obtenga de sus clientes limitar la naturaleza y el valor de los objetos a colocarse en las cajas de seguridad, aparece en contraste con las exigencias de una equilibrada adecuación de los intereses, que debe tener todo bien ordenado 
El Tribunal de Milán con sentencia $14.09 .89^{32}$, ha afirmado, ex Art. $1229^{\circ} \mathrm{cc}$, la nulidad del Art. $2^{\circ} \mathrm{nbu}$, considerándolo limitativo de la responsabilidad del banco. Los principios que se desumen del dictum son los siguientes:

1. En el caso de dolo o culpa grave por culpa del banco que ocasione daños al usuario, no operan las cláusulas del contrato, por las cuales el cliente se obliga a no conservar en la caja cosas que exceden un determinado valor. ${ }^{33}$. Además, se crítica al sector de la doctrina que ve en la relación de proporcionalidad - correspectividad entre el mayor riesgo de la banco y el mayor canon debido por los clientes, el fundamento de la licitud de los Art. $2^{\circ}$ y $3^{\circ} \mathrm{nbu}$, por cuanto tal relación «está fuera del sinalagma del contrato disciplinado por el Art. $1839^{\circ} \mathrm{cc}^{34}$ y se sostiene que el servicio de custodia al cual se obliga el banco es independiente del valor de los bienes depositados en las cajas.

2. El Instituto de vigilancia, al cual el banco ha confiado la tarea de cuidar sus locales, responde del hecho ilícito de sus auxiliares, con respecto de los titulares de las cajas de seguridad desvalijadas; cuando exista culpa grave del mismo banco por no haber adoptado medidas idóneas para prevenir o hacer dificil la comisión del ilícito, se afirma la responsabilidad solidaria del banco y del instituto de vigilancia ${ }^{35}$. En efecto, se distingue la eficacia subjetiva de la cláusula de exoneración de responsabilidad denominada omnicomprensiva en las relaciones banco-instituto de vigilancia, por cuanto ésta no puede ser opuesta a los terceros-usuarios, de su validez objetiva entre las partes ${ }^{36}$.

ordenamiento jurídico y no se presta a ser armónicamente integrada en la tabla de valores del ordenamiento» (Il corr. giur., cit.).

32 En Foro. it., 1990, 1038, con nota de PonZanelLI, La «sfortunata circolazione delle clausole di esonero: il caso dclle cassette di sicurezza; NGCC, 1990, cit, , con nota di PuHaLI, Il corr. giur., 1989, 1303, con nota de P. Carbont.

33 Se sanciona que: "La cláusula en cuestión no es nula por si, sino sólo en el caso concreto en el cual, se acierte que la violación de la integridad de la caja es debida a dolo o culpa grave del banco, (no por culpa leve de ésta), la cláusula limitativa de la responsabilidad opera y, por consiguiente, la banca deberá resarcir el daño sufrido por el cliente sólo en el limite convencionalmente establecido" (TRIB. Milavo, en Foro it., cit. 1047).

34 TRIB. MILANO, cit., 1048.

35 TRIB. MILANO, cit., 1038.

36 Así, Ponzanklli, Op. cit., 1041. En este sentido, también Niciro, "Statuti delle banche e Funzione bancaria rischio e responsabilità della banca, clausole di limitazione della responsabilità", en, a cura de Macciarone y Nigro, Gruffrè, Milano, 1981, que sostiene: «sea afirmando el valor normativo de los estatutos, sea negándolo, la 
En el ámbito de esta orientación jurisprudencial, se han pronunciado nuevamente el Tribunal de Milán con sentencias del $18.01 .90^{37}$ y del $04.06 .90^{38}$ y el Tribunal de Roma con sentencia de fecha $05.10 .90^{39}$. La misma posición ha sido confirmada por la Corte de Casación, con sentencias del 07.05 .92 , № $5421^{40}$ y del 12.05 .92 , № $5617^{41}$. La primera decisión enuncia estas directivas:

1. El contrato de cajas de seguridad es un contrato consensual, símil a la locación de cosas y a la locatio operis, en el cual el banco a través de la contraprestación de un canon, asume las siguientes obligaciones típicas:

a) conceder en uso locales idóneos para la prestación del servicio;

b) proveer a la custodia de los locales mismos y

c) tutelar la integridad exterior de las cajas.

2. El objeto del contrato es revisado en la seguridad de los mismos locales de los bancos en los cuales las cajas están situadas.

3. Las prestaciones a las cuales está obligado el banco consisten «esencialmente en un hacer teniendo como exclusivos términos de referencia los locales en cuestión».

conclusión, por lo que respecta a las disposiciones estatutarias limitativas del riesgo, termina por ser la misma: estas son inidóneas para incidir a nivel de relación contractual con los terceros; al máximo, se les puede reconocer el valor de condiciones generales de contrato" (cit., 124).

37 TRIB. Milano, 18.01.90, en BBTC, 1991, II, 765, con nota de SEPF, cit., quien afirma que la «declaración de valor» no debería considerarse como una obligación de los clientes, sino como una mera comunicación ofrecida al banco para fines estadísticos, "en tal caso se pondría el problema de la facultatividad y eventual sanción de tal información, cuando no sea exacta (SFPE, cit. 777).

38 TRIB. Milano, 04.06.90, en Giur. Merito, 1992, 54.

3.) TRIB. Roma, 05.10.90, en BBTC, II, 1993, 461, con nota de Contino, Responsabilità della banca per la custodia dei beni immesi in cassette di sicurezza.

40 Corte de Ocasión, 07.05.92, n. 5421, en BBTC, 1993, II, 363, con nota de SALANITRO, La responsabilità della banca nel servizio delle cassette di sicurezza, el cual afirma que si el daño es producido por incumplimiento de los auxiliares, «la exoneración preventiva de la responsabilidad por culpa o dolo debería ser admisible» (cit, 387) y en Foro it., No. 3, marzo, 1993, 879, con nota de CARINGillda, L'art. 2 delle norme bancarie uniformi in Cassazione: niente di nuovo sul fronte delle cassette di sicurezza, que sobre la base de los principios que inspiran el análisis económico del derecho, afirma que «la realización de una equilibrada ponderación comparativa de los costos y beneficios y, por ende, la misma sobrevivencia del servicio de quo, encuentran un serio obstáculo en el secreto del contenido de la caja” (cit., 887).

41 Corte de Ocasión, 12.05.92, N. 5617, en BBTC, 1993, II, 363. 
4. La prestación debe ser realizada con el máximo grado de diligencia correspondiente a la profesionalidad del bonus argentarius, lo que se concreta en la predisposición de los medios idóneos, aptos a evitar eventos perjudiciales, pero previsibles.

5. El Art. $2^{o}$ nbu, no integra el objeto del contrato introduciendo una ulterior obligación al cliente, aparte de aquella primaria de pagar el canon, sino tiene la única función de limitar la responsabilidad del banco; dicho pacto es válido en el caso de culpa leve, cual expresión de autonomía contractual, no contraria a las normas imperativas, pero en el caso de dolo o culpa grave contrasta con el principio de orden público al que se refiere el Art. $1229^{\circ} \mathrm{cc}$.

6. En razón de carácter presuntivo de la responsabilidad bancaria por el servicio de las cajas de seguridad, como se deduce, sea de la disciplina del código, sea de las normas bancarias uniformes, el cliente debe limitarse a la demostración del an y del quantum del daño sufrido, incumbiendo, en cambio, sobre el banco, la carga probatoria relativa a la presencia del caso fortuito.

Esta orientación, que parte de la premisa que la obligación del cliente de no colocar en la caja bienes de valor superior al pactado es extraño al contenido del tipo contractual, ha sido criticada porque importaría un contrasentido ${ }^{42}$, con el resultado que el banco estaría obligado «a contratar a oscuras $»^{43}$. No obstante a ello, de reciente, la Casación, con sentencia del 01.07.94, № 6225, ha confirmado la nulidad de los pactos que limitan el monto de los valores a conservar en la caja ${ }^{44}$.

En sentido contrario, se ha pronunciado el Tribunal de Roma, con sentencia del 27.04.90 y la Corte de Apelación de Roma con sentencias de fecha 06.03.90, 28.09.90 y $17.12 .9 \mathrm{I}^{45}$, que han considerado al Art. $2^{\circ}$ nbu como una cláusula delimitativa del contenido del contrato. La misma Corte de Apelación con sentencia del 06.11.90, ha completado

\footnotetext{
42 Así, Carrngelia, en cuanto: «del examen de la fisionomía completa del servicio $y$, específicamente, de la señalada inaccesibilidad de los bienes introducidos en institución de crédito, se deduce que la función práctica del contrato se manifiesta en la atribución al cliente-consumidor de la disponibilidad del contenido, de por si fluctuante, de la cajaexcluida toda ingerencia o control del banco-, con predisposición, por obra del argentarius, de la idónea organización ambiental y adecuados medios de protección externos» (cit. 890).

4.3 Caringilla, Op. cit., 891.

44 Corte de Ocasión, 01.07.94, n. 6225, en Il corr. giur., 1994, 967, con nota de V. Carbont.

45 Para esta orientación cfr. infra n. 10.
} 
tal afirmación afirmando que: «en caso de depósito de valores que excedan el límite pactado preventivamente, lo que no está garantizado es la restauración por equivalente en el caso de sustracción delictuosa, para lo cual está previsto en el ordenamiento jurídico otro tipo de contrato (seguros) que comporta, sin embargo, la renuncia del privilegio del secreto que está, en cambio, asegurado por el servicio de las cajas de seguridad» ${ }^{46}$.

\section{El caso fortuito y los criterios para determinar el quantum de las pretensiones resarcitorias de los usuarios dañados}

Habíamos ya evidenciado que el banco no está obligado resarcir al usuario cuando se demuestre que el daño ha sido originado por caso fortui$\mathrm{to}^{47}$. De ello la consecuencia que no es suficiente, a efectos que el banco sea exonerado de responsabilidad, la demostración de la mera ausencia de culpa. El c.c. hace recurso también a otras causas de limitación de la responsabilidad por incumplimiento, como la fuerza mayor ${ }^{48}$ y la causa no imputable ${ }^{49}$. Se ha subrayado que la doctrina más reciente considera por demás superada la distinción entre fortuito y fuerza mayor, mientras se va afirmando la tendencia a diferenciar el caso fortuito de la causa no imputable ${ }^{50}$. Se considera que el primero debe considerarse como un límite de responsabilidad «agravado» respecto a aquel expresado en términos de «causa no imputable» ${ }^{51}$. En efecto, esta última expresa un concepto de carácter prevalecientemente subjetivo, mientras el caso fortui-

4h Corte de Apelación Roma, 06.11.90, en Giust. cip., 1991, II, 2451.

47 Además del Art. $1839^{\circ}$ (cajas de seguridad), el c.c. ita. en materia contractual, hace referencia al caso fortuito en los arts. 1693 (responsabilidad por pérdida y avería), 1694 (presunciones de fortuito), 1787 (responsabilidad de los almacenes generales) y 1805 (perecimiento de la cosa en el comodato).

48 Considerada respecto al caso fortuito como una mera variación terminológica (CoTTINo, L'impossibilità soprapvenuta della prestazione e la responsabilità del debitore, Milano, 1955, 218). Es utilizada en materia de familia (art. 132 c.c.) haciendo reaparición en el art. 1785, reformado por el art. 3 de la ley del 10.06.78, n. 316 , de ratificación y ejecución de la Convención europea sobre la responsabilidad de los albergadores, firmada en París el 17.12.62 y que entró en vigor el 12.08.79.

49) Arts. 1218 y 1256 c.c.

50) Alpa, Op. cit., 383.

s1 Rlalmonte, Caso fortuito e forza maggiore, in Dig. Disc. Priv., Sez. Civ., II, Utht, Torino, 1988, 255. En este sentido, Castana, cuando afirma que: ula simple "causa no imputable», no exime al deudor de responsabilidad en las hipótesis en las cuales la norma prevea expresamente como eximente el "caso fortuito»" (Casu fortuito $t$ 
to, comporta una valoración de tipo objetivo, o sea extraña al deudor, «refiriéndose a un evento que proviene generalmente, del exterior de la persona del deudor y de su organización económica (hacienda)» $»^{52}$. Esta última cuestión, de otro lado, ha sido contestada por una parte de la doctrina que atribuye también al fortuito un carácter subjetivo ${ }^{53}$.

En materia de cajas de seguridad la jurisprudencia ha sido vacilante al comprender en el concepto de caso fortuito una serie de sucesos como, por ejemplo, la apertura forzada de cajas de seguridad de clientes israelíes, por parte de las autoridades alemanas o de la República Social ${ }^{54}$. Las mismas perplejidades se mantuvieron en materia de aluviones ${ }^{55}$. Con respecto a la rapiña y al hurto, estos pueden ser considerados como caso fortuito cuando se verifique tanto la imposibilidad de prevenirlos, aún con el uso de diligentes cautelas, como la lógica y razonable impredecibilidad de los mismos ${ }^{56}$. No obstante, en la mayoría de los casos, ha sido afirmada la responsabilidad del banco ${ }^{57}$. El incendio,

cassette di sicurezza, en Responsabilitì contrattuale ed extracontrattuale delle banche, cit, 103).

52 Castana, op. cit., 101 . En este sentido se considera que la causa no imputable, respecto del caso fortuito tiene «un significado menos riguroso y más amplio y por decir así, menos fatalista» (ALpA, Responsabilitò civile e danno, cit., 298).

53 En la elaboración doctrinal anterior al código civil de 1942, CovielLo defendía la concepción subjetiva y Polacco y VIVANTt aquella objetiva. La tesis subjetiva tuvo más fortuna bajo el código abrogado, la cual era debido a su difusión entre los comercialistas. OSTI, diferenciaba entre la imposibilidad objetiva y la subjetiva y se percibe en el actual código civil su infuencia. En este contexto: "La tendencia dominante era en el sentido de identificar el caso fortuito con la falta de culpa, pero también de considerar esencial en la elaboración de la teoría de caso fortuito, como causa libratoria, el elemento objetivo de la imposibilidad de la prestación (VISINTIN!, Inadempimento e mora del debitore, artt. 1218-1222, en Cod. Civ. Comm. diretto da S(HLfisingter, Giufrer:, Milano, 1987, 89).

54 En sentido positivo: Trib. Perugia, 13.12.46, en BBTC; AP. Ginova, 14.02.50, $i v i, 1950, \mathrm{II}, 352$ y AP. Torrno, 04.07.51, ivi, 1951, II, 388, con nota de Risci(ivo, Fortuito, causa non imputabile e diligenza. En sentido negativo, aunque en estos casos no se trataba de clientes israelitas, Trib. Piscara, 04.09.51, en BBTC, 1952, Il, 77; CAS, 13.10.55, ipi, 1956, II, 288, con nota crítica de Bunni, Forza maggiore in tema di servizio delle cassette di sicurezza.

55 En sentido positivo, AP. Firenze, 16.11.71, en Foro Pad., 1972, 317 y CAS., 27.07.76, en Giust. Cip., 1976, I, 1756. En sentido negativo, Trib. Finfinze, 24.04.70, en BBTC, II, 267 y Trib. Firknze, 11.03 .71 , cit.

56 Cas., 21.05.62, n. 1165, en Foro it, Mass., 1962, 359; Cas. 27.06.63, N. 1747, en Foro it. Rep., 1963, voz Danni per inadempimento, n. 8; AP. GENova, 21.02.80, en BBTC, 1980, II, 465.

57 TRIB. MILANo, 19.03.78, BBTC, 1978, II, 454; AP. ROMA, 07.10.80, ini, 1980, II, 477: Trib. Roma, 21.02 .83 , iwi, 1984, II, 99; TRIB. Napoli, 16.03.84, ivi, 
por último, puede constituir «caso fortuito» a menos que no se demuestre que el mismo ha tenido origen en los locales del banco ${ }^{58}$.

La jurisprudencia, al delimitar las notas características del caso fortuito, ha hecho recurso a los requisitos de inevitabilidad del hecho ${ }^{59}$, irresistibilidad $^{60}$, imprevisibilidad ${ }^{61}$ y extraordinariedad o excepcionalidad del hecho ${ }^{62}$. Sin embargo, se ha observado que «no es más característica connotante del fortuito el requisito de la impredecibilidad, porque pueden darse eventos predecibles absolutamente inevitables " ${ }^{63} \mathrm{y}$, además, que la jurisprudencia se dirige hacia una concepción objetiva y absoluta del caso fortuito ${ }^{64}$.

Tarea asaz dificil es aquella de determinar el quantum de un eventual resarcimiento cuando se demuestre la responsabilidad del banco. La carga de la prueba grava sobre el usuario, quien deberá demostrar el daño sufrido a causa de la sustracción o deterioro de los bienes custodiados en la caja. La jurisprudencia admite en estas hipótesis el recurso a presunciones simples ${ }^{65}$ (Arts. $2727^{\circ}$ y $2729^{\circ}$ c.c. ita.), así como la valoración equitativa del juez ${ }^{66}$ (Art. $1226^{0}$ c.c. ita). Por ejemplo, el Tribunal

1985, II, 506; Trib. Finknzr, 08.04.87, Giur. mer., 1988, I, 24; AP, Napoli, 28.04.87, en BBTC, 1989, II, 60; Trib. Roma, 26.11.87, en Corr. giur., 1988, 494, entre otras.

58 CAS., 07.03.76, n. 1129, en Foro it., 1976, I, 531.

5) Así, CAS., 29.03.76, n. 1 129, en BBTC, 1976, II, 173; CAS., 27.07.76, n. 2981, en NGCC, 1976, I, 1756; Trib. Roma, 15.02.78, en BBTC, 1978, II, 454, Trib. Roma, 27.01.82, en Giur. it., 1982, I, 2, 586.

(10) CAS., 27.07.76, n. 2981, cit, aunque equivocadamente con referencia a eventos extraordinarios y excepcionales (SEPE, op. cit., 773) y AP. Roma, 07.10.80, en BBTC, 1980, II, 477.

i) TRIB. Roma, 27.01.82, cit, AP. Firenze, 16.11.71, cit., CAS., 27.07.76, n. 2981, cit.

62 TRIB. MILAN(), 19.03.78, en BBTC, II, 454.

6. Alpa, Op. cit., quien da como ejemplo la sobrevenida de una catástrofe anunciada por el observatorio metereológico.

64 Alpa, Op. cit. En esta línea de pensamiento Visintini, cuando afirma que "el excesivo favor por la posición deudora expresada en las teorías subjetivistas choca contra el sistema legislativo y contra las tendencias de las sociedades evolucionadas y, por consiguiente, de los mercados evolucionados y racionales»(Op. cit., 135).

65. Así, TRIB. Pescara, 21.02.91: "La denuncia penal, suscrita por el contrayente del servicio bancario de las cajas de seguridad, que contiene la indicación de los bienes custodiados de la caja, aunque no asuma el rango de prueba civil, adquiere el valor de presunción con el carácter de verosimilitud" (en BBTC, 1992, II, 374).

to APP. Bari, 16.04.58, en BBTC, 1959, II, 203, con nota de MAJkLLo, Prove e presunzioni di danno in caso di effrazione di una cassetta di sicurezza; CORTE CAS., 13.05.82, n. 3000, ivi, 1984, II, 32, con nota de SALANITRo, Acceso di terzi, privi di autorizzazione scritta, a cassette di sicurczza: forma della ratifica; prova del contenuto 
de Milán, con sentencia de fecha $14.09 .89^{67}$, ha decidido en favor de los usuarios dañados, basándose principalmente en los resultados de las pruebas testimoniales ${ }^{68}$.

Frente a esta incertidumbre interpretativa, se va consolidando una suerte de policy ${ }^{69}$ que tutela a la persona en su perfil de consumidor, entendido en un sentido amplio. Para contrarrestar tal tendencia, los bancos han elaborado diversos mecanismos aptos a protegerlos en la eventualidad de un resarcimiento cuyo quantum es desconocido. Así, el seguro supletorio, que opera en la hipótesis en la cual el usuario quiera asegurar un importe superior a los límites máximos fijados. En tal caso, interviene una compañía de seguros, con una cobertura denominada «a primer riesgo absoluto", con la consecuencia que el eventual daño sufrido por el locatario será resarcido hasta la concurrencia del efectivo valor de los bienes sustraídos y/o destruidos y, obviamente, no más allá del límite máximo asegurado. Otras instituciones, en cambio, han introducido el denominado canon diferenciado, gestionado por el mismo banco sin la intervención de la compañía de seguros. Tal canon resulta de dos componentes, uno denominado fijo, vinculado al volumen de la caja y otro opcional, relacionado al mayor valor respecto a un límite establecido, de los bienes colocados que han sido declarados por el cliente, hasta un límite máximo.

Por el momento, los bancos italianos esperan que la A.B.I., emane nuevas disposiciones, a efectos de adoptar, en materia, un comportamiento común.

della cassetta: responsabilità del terzo e della banca, y en Giur. It, 1982, I, 1, 1413. Cfr. también PAOLETT, Il contratto di abbonamento alle cassette di sicurezza: note di dottrina e di giurisprudenza, en Mon. trib., 1968, 1484. En esta última se consideri que la valoración equitativa $e x$ art. 1226 c.c. ita. "es una valoración dirigida a acertar la cantidad a considerarse más probable, en base no sólo a las normas de común experiencia, sino también a todas las circunstancias del caso en examen".

67 Esta sentencia es distinta de aquella señalada en la nota 32, no obstante el dictum sea el mismo, las partes son diversas (en la ya comentada Dagnino y Montagna c. Banca Provinciale Lombarda y IVCM y ahora: Origgi, Vescovini y otros c. Banci popolare commercio e industria). En la primera sentencia se actuó la prueba testimonial, cosa que deja perplejo a PuHali «teniendo en cuenta que las operaciones realizadas por el usuario de la caja de seguridad, están cubiertas del secreto" (nota, cit., 343).

68 No obstante que entre los usuarios había una s.r.ltda. cuyos bienes depositados en la caja de seguridad no le pertenecían, sino a terceros (los socios de la misma), el Tribunal ha considerado que "la mera posición de "parte» en este contrato, basta para atribuir al usuario legitimación e interés para obrar con respecto del banco» (cit., 537).

69 Que está constituida por una serie de principios no explícitos o encubiertos, que son manifestaciones de una determinada política de derecho. Cfr. Alpa, I principi generali, en Tratt, Dir. Priv. a cura de Iulrica y ZatTl, Giufrré, 1993, 444. 


\section{5 .El modelo diseñado por el operador jurídico nacional en materia de alquiler de cajas de seguridad}

A falta de disposiciones administrativas emanadas por la Superintendencia de Banca y Seguros, los bancos peruanos han elaborado sus formularios de contrato de alquiler de cajas de seguridad, basándose en lo dispuesto por el código civil y la ley de bancos, en su momento. La derogada Ley General de Instituciones Bancarias, Financieras y de Seguros, D. Leg. No. 770 , del 28.10 .93 , en su Art. $357^{\circ}$, establecía que «los bancos quedan eximidos de responsabilidad por el alquiler de cajas de seguridad en los casos en que en ellas desaparezcan como consecuencia de catástrofes o incendios, así como cuando, habiendo adoptado razonables previsiones de seguridad, sean violentadas por acción delictiva de terceros perpetrada con amenaza grave para la vida o la seguridad de las personas». Este dispositivo fué criticado porque se advertía que podían presentarse situaciones en las cuales, habiéndose «adoptado razonables previsiones de seguridad", se fuercen las cajas de seguridad, sin que con ello, necesariamente, se haya atentado contra la vida o la seguridad de las personas ${ }^{70}$. La actual Ley General del Sistema Financiero y del Sistema de Seguros y Orgánica de la Superintendencia de Banca y Seguros, Ley No. 26702, del 06.12.96, no contempla un tenor similar, limitándose a incluír, en su Art. $221^{\circ}$, inc. 33, dentro de las operaciones y servicios que pueden realizar las empresas del Sistema Financiero, la de «dar en alquiler cajas de seguridad».

Es usual leer el siguiente tenor dentro de los formularios bancarios que contienen el contrato de alquiler de cajas de seguridad: «el banco garantiza únicamente la integridad exterior de la caja de seguridad que alquila, eximiéndose de toda otra responsabilidad.

Igualmente, el banco no se hace responsable por daños y/o perjuicios que puedan provenir de daños causados por casos fortuitos o de fuerza mayor, limitándose su responsabilidad a aquellos provenientes de dolo, culpa o negligencia inexcusables de acuerdo al Art. $1321^{\circ}$ del c.c. y por el valor estrictamente económico de los bienes que efectivamente se hayan depositado en la caja de seguridad, hasta un máximo de 5 Ingresos Mínimos Vitales de la Provincia de Lima».

El hecho de limitar al quantum resarcitorio en caso de dolo, culpa o negligencia inexcusables, colisiona con lo dispuesto por el Art. $1328^{\circ}$ c.c.,

70) Casthllares Agullar, Perú. Informe Económico Trimestral, Banco de Crédito, diciembre 1993, 26, quien hace referencia a un caso ocurrido en un banco colombiano. 
el cual sanciona con nulidad «toda estipulación que excluya o limite la responsabilidad por dolo o culpa inexcusable del deudor o de los terceros de quien éste se valga». Es por ello que dicha cláusula contractual, salvo para el caso de culpa leve (como sucede en el modelo italiano), carece de efecto jurídico alguno. 